\title{
Research Article \\ Energy Optimal Control Strategy of PHEV Based on PMP Algorithm
}

\author{
Tiezhou Wu, Yi Ding, and Yushan Xu \\ Hubei Collaborative Innovation Center for High-Efficiency Utilization of Solar Energy, Hubei University of Technology, \\ Wuhan 430068, China \\ Correspondence should be addressed to Yi Ding; 191593927@qq.com
}

Received 7 September 2016; Revised 2 January 2017; Accepted 15 January 2017; Published 28 February 2017

Academic Editor: Yongji Wang

Copyright (C) 2017 Tiezhou Wu et al. This is an open access article distributed under the Creative Commons Attribution License, which permits unrestricted use, distribution, and reproduction in any medium, provided the original work is properly cited.

Under the global voice of "energy saving" and the current boom in the development of energy storage technology at home and abroad, energy optimal control of the whole hybrid electric vehicle power system, as one of the core technologies of electric vehicles, is bound to become a hot target of "clean energy" vehicle development and research. This paper considers the constraints to the performance of energy storage system in Parallel Hybrid Electric Vehicle (PHEV), from which lithium-ion battery frequently charges/discharges, PHEV largely consumes energy of fuel, and their are difficulty in energy recovery and other issues in a single cycle; the research uses lithium-ion battery combined with super-capacitor (SC), which is hybrid energy storage system (Li-SC HESS), working together with internal combustion engine (ICE) to drive PHEV. Combined with PSO-PI controller and Li-SC HESS internal power limited management approach, the research proposes the PHEV energy optimal control strategy. It is based on revised Pontryagin's minimum principle (PMP) algorithm, which establishes the PHEV vehicle simulation model through ADVISOR software and verifies the effectiveness and feasibility. Finally, the results show that the energy optimization control strategy can improve the instantaneity of tracking PHEV minimum fuel consumption track, implement energy saving, and prolong the life of lithium-ion batteries and thereby can improve hybrid energy storage system performance.

\section{Introduction}

In 2015, the incident that the illegal use of emissions control software of Volkswagen further triggers a strong concern on cars "energy saving" problem. So it is essential to use energy storage technologies to alleviate energy waste. Considering the key factor that restricts battery performance and service life in lithium-ion battery, this paper applies Li-SC HESS to PHEV. However, how to coordinate each of storage units charging/discharging and optimize energy management of vehicle power system in real time is the key to implement the performance optimization of Li-SC HESS and minimize fuel consumption in PHEV. Santucci et al. [1] proposes a new dynamic optimization method by combining model predictive control with DP algorithm and considering the state of charge (SOC) each of SC and lithiumion battery, together with simplified battery aging models and others. Masih-Tehrani et al. [2] considers factors like fuel consumption, periodic battery replacement, and others and proposes a DP algorithm based on PHEV management costs, which considerably improves energy economy. But DP algorithm has a larger calculation and is more timeconsuming, so it is difficult for practical application, while the energy optimal control strategy based on PMP algorithm is becoming research focus in recent years with the advantages of its fast speed and the smaller amount of calculation than DP algorithm.

In this paper, PMP global optimization control algorithm has been adopted, which combines with Li-SC HESS internal power limited management strategy, thus taking an energy optimal control to PHEV, having an optimal management of lithium-ion battery charging/discharging states, improving the Li-SC HESS performance, and meanwhile ensuring that the vehicle fuel consumption during running can track the fuel consumption minimum trajectory in real time.

\section{PHEV Driven Model}

2.1. Vehicle Structure of PHEV. According to vehicle powertrain structure, HEV can be divided into SHEV, PHEV, and 


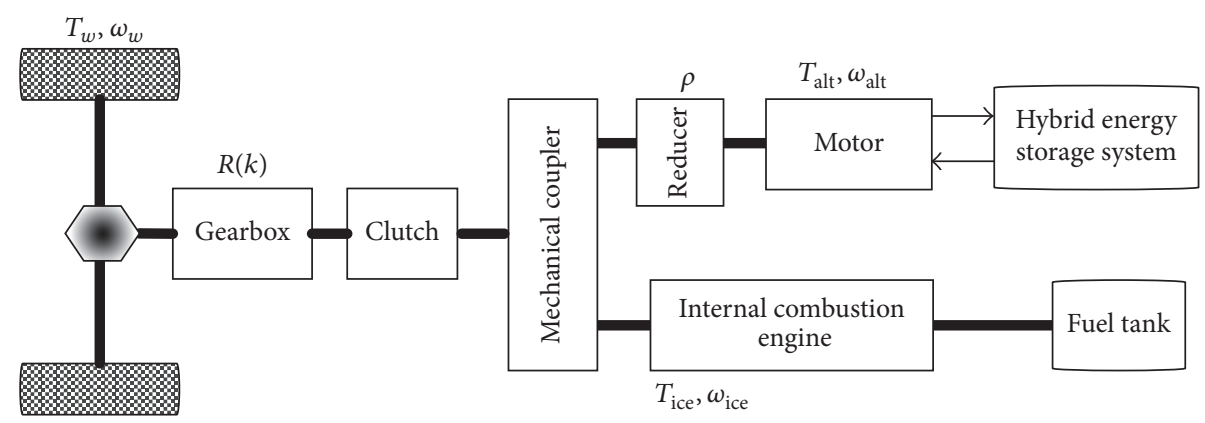

Figure 1: Vehicle Structure of PHEV.

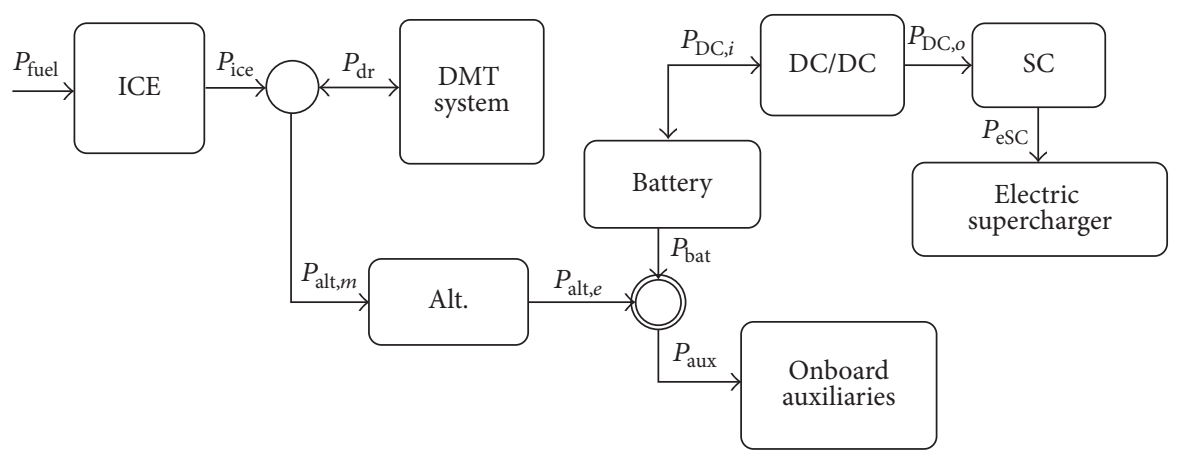

Mechanical power flow nodes
Electric power flow nodes
DMT: dynamic mechanical transmission

FIgURE 2: Power flow schematic of PHEV power system.

S-PHEV, wherein the SHEV control system is not considered because of its relatively simple control and its large energy conversion, which easily poses a threat to lithium-ion batteries life. PHEV can make energy more efficient and fuel economy relatively higher, so in order to reduce fuel consumption as much as possible this paper selects $\mathrm{PHEV}$ powertrain structure, with its power-type composition architecture being shown in Figure 1, and the corresponding power flows of PHEV power system are shown in Figure 2. Its main components include gearbox, clutch, mechanical coupling, reducer, alternator, ICE, fuel tank, hybrid energy storage system, and other modules. The vehicle is equipped with a 5-speed manual gearbox; the AC alternator is connected to ICE which owns a fixed speed ratio, to reduce the weight and volume of mechanical transmission and simplify the structure.

From Figure $1, T_{w}, T_{\text {ice }}$, and $T_{\text {alt }}$ are, respectively, the wheels, ICE, and alternator torque, Nm; $\omega_{w}, \omega_{\text {ice }}$, and $\omega_{\text {alt }}$ are the wheels, ICE, and alternator speed, rpm; $\eta_{\mathrm{gb}}$ is the gearbox efficiency; $k$ is the number of gearbox's gears that corresponds to its gear ratio $R(k) ; Q_{l h v}$ is fuel energy density, $\mathrm{kJ} / \mathrm{kg}$.

The vehicle power flow of PHEV power system is shown in Figure 2, the bidirection arrow indicates energy flow being bidirectional. Under the power-train operating mode in PHEV drive cycle, one part of power comes from energy conversion of ICE; the other part is derived from Li-SC HESS power driving. Considering the constraints of traffic environment and road situation, the electric vehicles are often in the state of standstill, started, acceleration or smooth driving, and deceleration. If lithium-ion batteries and SC output power can be allocated reasonably, Li-SC HESS can effectively reduce fuel consumption and improve PHEV economic performance. When vehicle starts, in order to reduce energy consumption of ICE, SC provides high instantaneous electric power by electric supercharging device firstly, reducing lithium-ion lifetime impact because of its high current discharging. When PHEV drives in steady, lithium-ion batteries, as the main power of Li-SC HESS, supply the portion of the power to alternator. When the vehicle accelerates or drives uphill, in order to meet the demand of high power portion, flat lithium-ion battery charging/discharging process, SC fills up the vacancy of alternator power gaps peak change preferentially. When the vehicle brakes or drives downhill, SC and lithium-ion batteries recycle braking energy at the same time, and SC takes priority to recycle the energy of high current power at the beginning.

2.2. Li-SC HESS Model. As PHEV energy storage device, Li-SC HESS owns its control module which is also one 


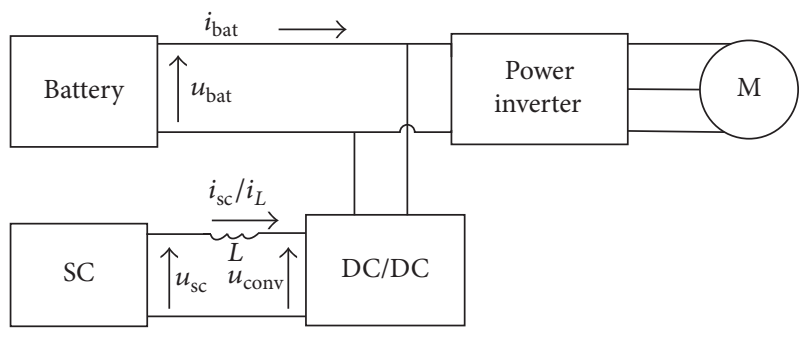

FIgUre 3: The topology of Li-SC HESS.

part of the whole vehicle control. Selecting Li-SC HESS appropriate equivalent circuit model, which includes its topology, the equivalent circuit model of each storage element, and control variables, is the precondition for the study on PHEV power system energy optimization control strategy. When the vehicle is in state of accelerating or moving uphill, this structure can quickly respond to the demand of alternator's high instantaneous power and it can also recover energy when the vehicle is in state of decelerating and braking. Besides, with respect to the topology structure between lithium-ion batteries and SC, each Li-SC HESS topology will make a difference in its component parts, different practical applications, and different connections, like the 4 kinds as follows: SC and lithium batteries parallel directly, SC and lithium-ion batteries parallel through each independent DC/DC converter, lithium-ion batteries (/SC) connects to DC/DC, and then parallel with SC (/lithium-ion batteries). Considering the advantages of HESS combination type, energy conversion efficiency, the complexity of system structure, and others, SC connects to DC/DC converter and then parallels with lithium-ion batteries (Figure 3), which is more suitable for the requirements of current dynamics and the low cost of PHEV. Compared with the independently configured DC/DC converters in parallel, its control method is relatively simpler, and the efficiency is relatively higher, which can better meet the needs of reducing vehicle fuel consumption in this paper.

2.2.1. Lithium-Ion Battery Equivalent Model. This paper selects first-order RC parallel circuit as lithium-ion battery equivalent model (Figure 4), due to its low cost and less calculation; now the model has been accepted by some lithium-ion battery businesses, such as Sony and Panasonic.

Under different road conditions, the current will change dramatically with different power demands of the alternator; environmental factors such as battery temperature will also be affected, thus forming a threat to the lithium-ion battery life and safety. From Figure 4, lithium-ion battery state of charge $\mathrm{SOC}_{\mathrm{bat}}(t)$ is represented as follows:

$$
\begin{aligned}
& \operatorname{SOC}_{\text {bat }}(t) \\
& =\frac{1}{Q_{\text {bat }, 0}} \int_{0}^{T} \alpha\left(I_{\text {bat }}(t)\right) \beta\left(T_{\text {bat }}(t)\right) I_{\text {bat }}(t) d t \\
& \quad+\operatorname{SOC}_{\text {bat }, 0},
\end{aligned}
$$

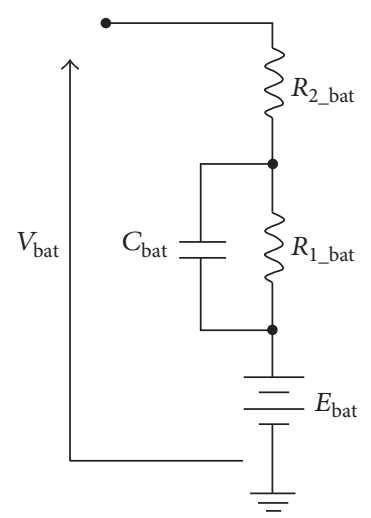

FIGURE 4: Lithium-ion battery equivalent model based on first-order RC parallel circuit.

$$
\begin{aligned}
m \cdot c_{p} & \cdot \frac{d T_{\text {bat }}(t)}{d t} \\
= & I_{\text {bat }}(t)^{2} \cdot R_{2 \_ \text {bat }} \\
& +\frac{1}{R_{1 \_ \text {bat }}}\left[V_{\text {bat }}(t)-E_{\text {bat }}(t)-R_{2 \_ \text {bat }} I_{\text {bat }}(t)\right]^{2} \\
& -h_{c} A\left[T_{\text {bat }}(t)-T_{a}\right] .
\end{aligned}
$$

From (1), $Q_{\text {bat.0 }}$ is battery capacity; $\alpha\left(I_{\text {bat }}(t)\right)$ and $\beta\left(T_{\text {bat }}(t)\right)$ are battery impact factor of charging/discharging rate and thermal effect, which, respectively, represent the battery current $I_{\mathrm{bat}}(t)$ and temperature $T_{\mathrm{bat}}(t)$; $\mathrm{SOC}_{\mathrm{bat}, 0}$ is the initial value of battery state of charge (SOC); $m$ stands for the battery quality; $c_{p}$ represents battery's specific heat capacity; $V_{\text {bat }}$ is the terminal voltage of batteries' equivalent model; $h_{c}$ means the convective heat transfer coefficient; $A$ is the equivalent surface area; $T_{a}$ is battery's ambient temperature.

From (1), $\mathrm{SOC}_{\text {bat }}(t)$ is related to $T_{\text {bat }}(t)$ and $I_{\text {bat }}(t)$ in the current time, as $T_{\text {bat }}(t)$ is a function of $I_{\text {bat }}(t)$. So SOC $C_{\text {bat }}(t)$ can be eventually represented by $I_{\text {bat }}(t)$, as with formula (2) as follows:

$$
\begin{aligned}
\operatorname{SOC}_{\text {bat }}(t)= & \frac{1}{Q_{\text {bat }, 0}} \int_{0}^{t_{\text {sim }}} \sigma\left(I_{\text {bat }}(t)\right) I_{\text {bat }}(t) d t \\
& +\operatorname{SOC}_{\text {bat }, 0} \Longrightarrow \\
\operatorname{SOC}_{\text {bat }}(t)= & -\frac{I_{\text {bat }}\left(\operatorname{SOC}_{\text {bat }}(t)\right)}{Q_{\text {bat }, 0}} \\
\operatorname{SOC}_{\text {bat }}(0)= & \operatorname{SOC}_{\text {bat }, 0} .
\end{aligned}
$$

Similarly, the instantaneous power $P_{\text {bat }}(t)$ and the corresponding current $I_{\text {bat }}\left(\mathrm{SOC}_{\text {bat }}(t)\right)$ are shown in formula (3), wherein $R_{\text {bat }}$ is the battery equivalent resistance.

$$
\begin{aligned}
P_{\text {bat }}(t) & =R_{\text {bat }} I_{\text {bat }}^{2}(t)+E_{\text {bat }} I_{\text {bat }}(t), \\
I_{\text {bat }}(t) & =\frac{E_{\text {bat }}-\sqrt{E_{\text {bat }}^{2}-4 R_{\text {bat }} P_{\text {bat }}(t)}}{2 R_{\text {bat }}} \\
R_{\text {bat }} & =R_{1 \_ \text {bat }} / / C_{\text {bat }}+R_{2 \text { _bat }} .
\end{aligned}
$$




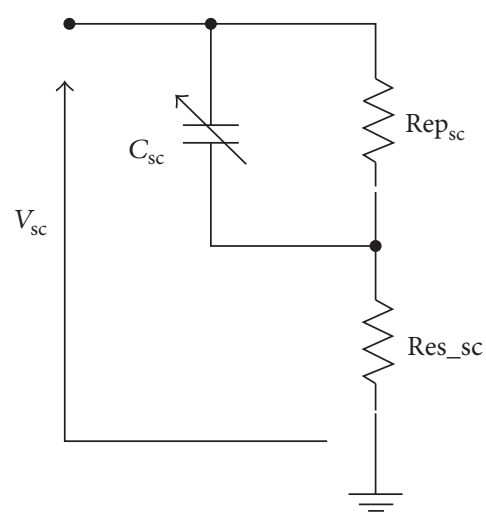

FIGURE 5: SC equivalent model based on first-order RC parallel circuit.

2.2.2. SC Equivalent Model. Similarly, considering the structural flexibility and lower computing costs, choosing the equivalent first-order RC parallel circuit structure as SC equivalent model, like Figure 5, the physical significance of each parameter is clear enough. Under the PHEV power systems complex conditions, this model can well describe large current and voltage fluctuations, which also has a relatively high degree of practical engineering.

From Figure 5, the voltage $V_{\mathrm{sc}}(t)$ of $C_{\mathrm{sc}}$ is similar to $V_{\mathrm{bat}}(t)$ above; the expression $\mathrm{SOC}_{\mathrm{sc}}(t)$ of SC, instantaneous power $P_{\mathrm{sc}}(t)$, and the current $I_{\mathrm{sc}}\left(\mathrm{SOC}_{\mathrm{sc}}(t)\right)$ are shown in formula (4) as follows:

$$
\begin{aligned}
& \operatorname{sOC}_{\mathrm{sc}}(t)=\frac{d \mathrm{SOC}_{\mathrm{sc}}(t)}{d t}=\frac{d\left(V_{\mathrm{sc}}(t) C_{\mathrm{sc}} / V_{\mathrm{sc}, \max } C_{\mathrm{sc}}\right)}{d t} \\
& =\frac{\mathrm{SOC}_{\mathrm{sc}}(t) V_{\mathrm{sc}, \max }-\sqrt{\left(\mathrm{SOC}_{\mathrm{sc}}(t) V_{\mathrm{sc}, \max }\right)^{2}-4 R_{\mathrm{sc}} P_{\mathrm{sc}}(t)}}{2 R_{\mathrm{sc}} V_{\mathrm{sc}, \max } C_{\mathrm{sc}}}, \\
& \operatorname{SOC}_{\mathrm{sc}}(0)=\operatorname{SOC}_{\mathrm{sc}, 0} \\
& P_{\mathrm{sc}}(t)=R_{\mathrm{sc}} I_{\mathrm{sc}}^{2}\left(\mathrm{SOC}_{\mathrm{sc}}(t)\right)+V_{\mathrm{sc}} I_{\mathrm{sc}}\left(\mathrm{SOC}_{\mathrm{sc}}(t)\right) \\
& I_{\mathrm{sc}}\left(\operatorname{SOC}_{\mathrm{sc}}(t)\right) \\
& =\frac{\operatorname{SOC}_{\mathrm{sc}}(t) V_{\mathrm{sc}, \max }-\sqrt{\left(\mathrm{SOC}_{\mathrm{sc}}(t) V_{\mathrm{sc}, \max }\right)^{2}-4 R_{\mathrm{sc}} P_{\mathrm{sc}}(t)}}{2 R_{\mathrm{sc}}} .
\end{aligned}
$$

Where $R_{\mathrm{sc}}$ is SC equivalent resistance, which is composed of the equivalent circuit of $R_{\text {ep_sc }}$ paralleling with $C_{\mathrm{sc}}$ and then connecting with $R_{\text {es_sc. }} . V_{\text {sc, max }}$ is the maximum voltage of $V_{\mathrm{sc}}$. $P_{\mathrm{sc}}(t)$ is the instantaneous power of SC. $\mathrm{SOC}_{\mathrm{sc}, 0}$ is the initial value of SOC.

\section{Problem Description of PHEV Energy Management Optimization}

3.1. Selection of Control Objective Function. In response to the development goals of green energy economy, it should make better performance of Li-SC HESS, minimizing ICE energy consumption, reducing economic costs, and reducing environmental pollution. This paper makes ICE minimum energy consumption as a control target variable. Throughout the drive cycle $[0: T]$ of PHEV power systems, it assumes that ICE energy consumption function $Q_{c}$ is

$$
Q_{c}=\int_{0}^{T} P_{\text {fuel }}\left(T_{\text {ice }}(t), \omega_{\text {ice }}(t)\right) d t
$$

where $T$ is the end time in calculation, $P_{\text {fuel }}\left(T_{\text {ice }}(t), \omega_{\text {ice }}(t)\right)$ is the instantaneous fuel power at time $t$, and the corresponding speed and torque of ICE are $\omega_{\text {ice }}(t)$ and $T_{\text {ice }}(t)$.

3.2. Objective Function Control Variable Constraints. PHEV energy optimal management requires considering the restrictions of the objective function from a global point of view, mainly from vehicle structure constraints; both physical model constraints of ICE and alternator and Li-SC HESS state constraints.

3.2.1. Vehicle Structural Constraints. Designing the rational energy optimal control strategy usually regards the vehicle driving force and vehicle speed as the given system status conditions, which are denoted as $F_{v}(t) / \mathrm{N}$ and $v /(\mathrm{km} / \mathrm{h})$ and also are converted into wheel torque $T_{w}(t)$ and wheel speed $\omega_{w}(t)$ of backward system in driving period, for the convenience of description. That means being, respectively, converted into the superposition of $T_{\text {ice }}(t)$ and $T_{\text {alt }}(t)$ and $\omega_{\text {ice }}(t)$ and $\omega_{\text {alt }}(t)$ (the torque and speed of alternator output are $T_{\text {alt }}(t)$ and $\left.\omega_{\text {alt }}(t)\right)$. The specific relationship description is shown in formula (6).

$$
\begin{aligned}
T_{w}(t) & =R(k(t)) \eta_{\mathrm{gb}}\left(T_{\mathrm{ice}}(t)+\rho T_{\mathrm{alt}}(t)\right) \\
& =R(k(t)) \eta_{\mathrm{gb}} T_{\mathrm{ps}}(t), \\
\omega_{w}(t) & =\frac{\omega_{\text {ice }}(t)}{R(k(t))}=\frac{\omega_{\mathrm{alt}}(t)}{\rho R(k(t))} .
\end{aligned}
$$

From (6), $k(t)$ is the number of vehicle gear, the driving cycle is usually defined by $\omega_{w}(t)$ and $k(t)$. When $\omega_{w}(t)$ and $k(t)$ are already known, it is pretty easy to deduce the kinetics equation (5) of ICE fuel consumption function at the desired wheel torque $T_{w}(t)$.

\subsubsection{ICE and Alternator Physical Model Control Constraints.}

ICE is a complex system; many of its physical phenomena are not easy to be modeled, like the burning process. In this case, the research ignores the temperature dependence of ICE and its dynamic characteristics, and then get the distribution graph of instantaneous fuel consumption function under the action of $T_{\text {ice }}(t)$ and $\omega_{\text {ice }}(t)$ with the static look-up table (LUT) showing as Figure 6. Similarly, as given $T_{\text {alt }}$ and $\omega_{\text {alt }}$, combined with the relevant alternator LUT method, efficiency function of electric motor and the corresponding maximum current graph can be derived from Figures 7 and 8 .

As it can be seen from Figures 6-8, when ICE and alternator speed are given, the corresponding torques are 


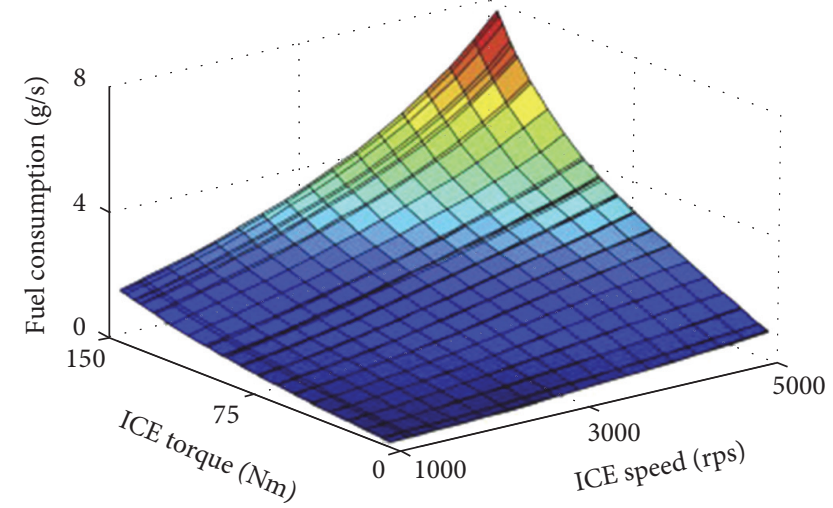

FIGURE 6: Distribution graph of instantaneous fuel consumption function under the action of $T_{\text {ice }}(t), \omega_{\text {ice }}(t)$.

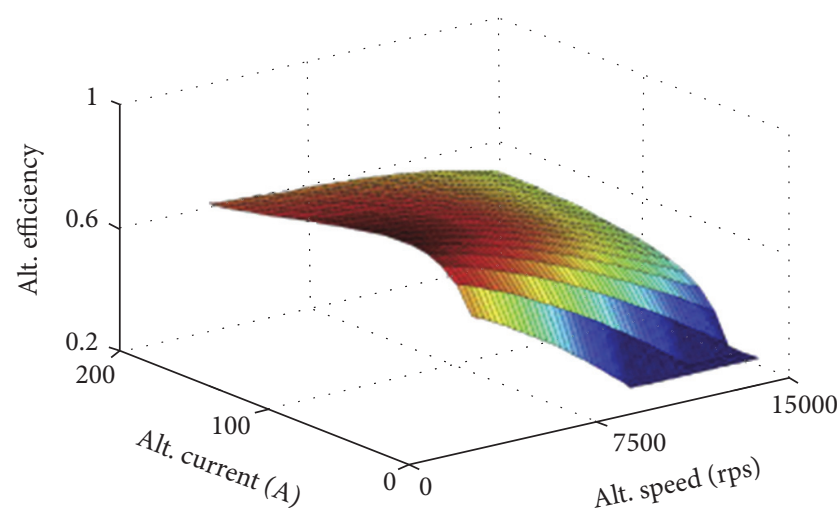

FIGURE 7: Graph of Alt. efficiency function curve under the action of $I_{\text {alt }}, \omega_{\text {alt }}$.

constrained by their maximum available torque. So formula (7) is defined as follows:

$$
\begin{aligned}
\omega_{\text {ice,min }} & \leq \omega_{\text {ice }}(t) \leq \omega_{\text {ice, } \max }, \\
T_{\text {ice,min }}\left(\omega_{\text {ice }}(t)\right) & \leq T_{\text {ice }}(t) \leq T_{\text {ice, } \max }\left(\omega_{\text {ice }}(t)\right), \\
\omega_{\text {alt,min }} \leq \omega_{\text {alt }}(t) & \leq \omega_{\text {alt, } \text { max }}, \\
T_{\text {alt,min }}\left(\omega_{\text {alt }}(t)\right) & \leq T_{\text {alt }}(t) \leq T_{\text {alt,max }}\left(\omega_{\text {alt }}(t)\right) .
\end{aligned}
$$

Formula (6) shows that the spindle torque $T_{\mathrm{ps}}(t)=$ $T_{\text {ice }}(t)+\rho T_{\text {alt }}(t)$, considering the alternator torque constraints, at any time $t$, both of the ICE minimum $T_{\text {ice,min }}\left(\omega_{\text {ice }}(t)\right)$ and maximum $\overline{T_{\text {ice, } \max }}\left(\omega_{\text {ice }}(t)\right)$ satisfies formula $(8)$.

$$
\begin{aligned}
& \frac{T_{\text {ice, } \min }}{}\left(\omega_{\text {ice }}(t)\right)=\max \left\{T_{\text {ice, } \min }\left(\omega_{\text {ice }}(t)\right), T_{\mathrm{ps}}(t)\right. \\
& \left.\quad-\rho T_{\text {alt,max }}\left(\omega_{\text {alt }}(t)\right)\right\} \\
& \overline{T_{\text {ice, } \max }}\left(\omega_{\text {ice }}(t)\right)=\min \left\{T_{\text {ice, } \max }\left(\omega_{\text {ice }}(t)\right), T_{\mathrm{ps}}(t)\right. \\
& \left.\quad-\rho T_{\text {alt,min }}\left(\omega_{\text {alt }}(t)\right)\right\} .
\end{aligned}
$$

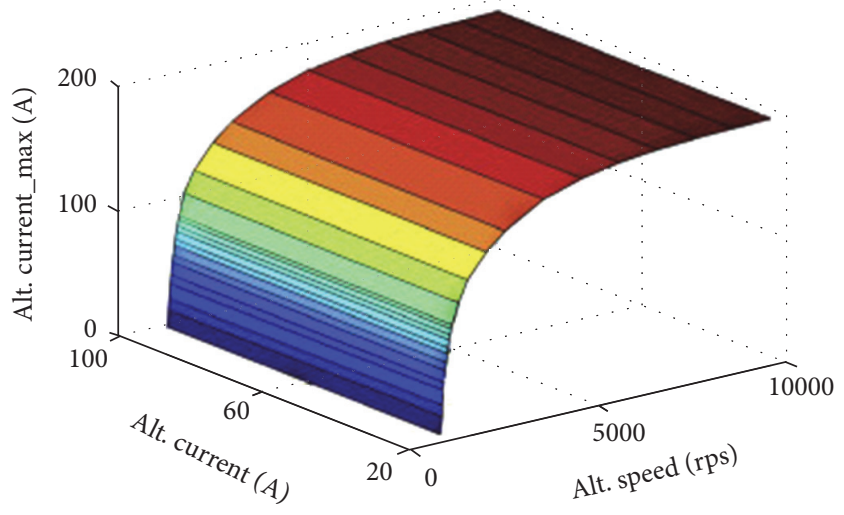

FIGURE 8: Graph of Alt. maximum current curve under the action of $I_{\text {alt }}, \omega_{\text {alt }}$.

3.2.3. Li-SC HESS Control Constraints. Among energy storage element, SOC is an important parameter of overcharging/overdischarging and cycle-life of storage elements. According to Li-SC HESS equivalent mathematical model, in order to minimize the charging/discharging times in the certain drive cycle, $\mathrm{SOC}_{\mathrm{bat}}(t)$ should be limited, and so it is the same with battery current $I_{\text {bat }}(t)$ during the charging/discharging process, and $\operatorname{SOC}_{\mathrm{sc}}(t), I_{\mathrm{sc}}(t)$ is shown in equation (9).

$$
\begin{aligned}
\mathrm{SOC}_{\mathrm{bat}, \text { min }} & \leq \mathrm{SOC}_{\mathrm{bat}}(t) \leq \mathrm{SOC}_{\mathrm{bat}, \text { max }}, \\
I_{\mathrm{bat}, \text { min }} & \leq I_{\mathrm{bat}}(t) \leq I_{\mathrm{bat}, \text { max }} \\
\mathrm{SOC}_{\mathrm{sc}, \text { min }} & \leq \mathrm{SOC}_{\mathrm{sc}}(t) \leq \mathrm{SOC}_{\mathrm{sc}, \text { max }} \\
I_{\mathrm{sc}, \text { min }} & \leq I_{\mathrm{sc}}(t) \leq I_{\mathrm{sc}, \text { max }} .
\end{aligned}
$$

Since lithium-ion battery and SC are all energy buffer devices, in the continued charging state, assessing fuel economy of the energy optimization control, PMP algorithm should meet the need of the end constraints conditions of $\Delta \mathrm{SOC}_{\text {bat }} \approx 0$ and $\Delta \mathrm{SOC}_{\mathrm{sc}} \approx 0$, that is, formula (10).

$$
\begin{gathered}
\Delta \mathrm{SOC}_{\mathrm{bat}} \triangleq \mathrm{SOC}_{\mathrm{bat}}(T)-\mathrm{SOC}_{\mathrm{bat}}(0), \\
\Delta \mathrm{SOC}_{\mathrm{sc}} \triangleq \mathrm{SOC}_{\mathrm{sc}}(T)-\mathrm{SOC}_{\mathrm{sc}}(0) .
\end{gathered}
$$

\section{Energy Optimal Management Strategy Based on PMP Algorithm}

4.1. Construction of Hamiltonian Function. With the consumption function $Q_{c}$, in formula (5), when the final time is given, energy consumption can be converted into a Lagrange problem with constrained terminal state, which corresponded to Hamilton function, as formula (11).

$$
\begin{gathered}
H_{a}\left(\mathrm{SOC}_{\mathrm{bat}}, \mathrm{SOC}_{\mathrm{sc}}, T_{\mathrm{ice}}, I_{\mathrm{DC}, o}, \lambda_{1}, \lambda_{2}\right) \\
=P_{\text {fuel }}\left(T_{\mathrm{ice}}, \omega_{\text {ice }}\right)-\lambda_{1} \frac{I_{\mathrm{bat}}\left(\mathrm{SOC}_{\mathrm{bat}}\right)}{\mathrm{Q}_{\mathrm{bat}, 0}} \cdots \\
\quad-\lambda_{2} \frac{I_{\mathrm{sc}}\left(\mathrm{SOC}_{\mathrm{sc}}, I_{\mathrm{DC}, o}\right)}{\mathrm{Q}_{\mathrm{bat}, 0}}+\lambda_{d} \Phi\left(\mathrm{SOC}_{\mathrm{sc}}\right) .
\end{gathered}
$$


From (11), In order to connect the dynamic characteristics of both lithium-ion battery and SC, this research considers SC characteristics that it has the priority to respond large current changes quickly and its protection to overcharge and overdischarge, and then it brings in a dynamic buffer variable $\Phi\left(\mathrm{SOC}_{\mathrm{sc}}\right)$ as a penalty function to restrain Li-SC HESS dynamic processes, which are described in the formula (12).

$$
\begin{aligned}
\dot{X}_{d} \triangleq & \Phi\left(\mathrm{SOC}_{\mathrm{sc}}\right) \\
= & {\left[\mathrm{SOC}_{\mathrm{sc}}-\mathrm{SOC}_{\mathrm{sc}, \min }\right]^{2} \mathrm{sg}\left(\mathrm{SOC}_{\mathrm{sc}, \text { min }}-\mathrm{SOC}_{\mathrm{sc}}\right) } \\
& +\left[\mathrm{SOC}_{\mathrm{sc}, \max }-\mathrm{SOC}_{\mathrm{sc}}\right]^{2} \mathrm{sg}\left(\mathrm{SOC}_{\mathrm{sc}}-\mathrm{SOC}_{\mathrm{sc}, \text { max }}\right) .
\end{aligned}
$$

From $(12), \operatorname{sg}(x) \triangleq\{0, x<0 ; 1, x \geq 0\}, \dot{X}_{d}(t) \geq 0$, $\forall t \in[0, T]$, only if $\mathrm{SOC}_{\mathrm{sc}}$ satisfies formula (12); it should be $\dot{X}_{d}(t)=0$. There, $X_{d}(t)=\int_{0}^{t} \dot{X}_{d}(t) d t+X_{d}(0)$ and its terminal constraint condition is $X_{d}(T)=X_{d}(0)=0$.

4.2. Solution of Extreme Value of Li-SC HESS Output Coefficient. According to Hamiltonian function equation (11), this research seeks these necessary conditions for the minimum value of $Q_{c}$, that is, a set of costate equations (formula (13)), for the sake of solving the initial value of these costate variables.

$$
\begin{aligned}
& \mathrm{SOCC}_{\mathrm{bat}}^{*}=\frac{\partial H_{\alpha}(\cdot)}{\partial \lambda_{1}}=-\frac{I_{\mathrm{bat}}\left(\mathrm{SOC}_{\mathrm{bat}}^{*}\right)}{Q_{\mathrm{bat}, 0}}, \\
& \dot{\lambda}_{1}^{*}=-\frac{\partial H_{\alpha}(\cdot)}{\partial \mathrm{SOC}_{\mathrm{bat}}}=\frac{\lambda_{1}^{*}}{Q_{\mathrm{bat}, 0}} \frac{\partial I_{\mathrm{bat}}\left(\mathrm{SOC}_{\mathrm{bat}}^{*}\right)}{\partial \mathrm{SOC}_{\mathrm{bat}}}, \\
& \mathrm{SOCC}_{\mathrm{sc}}^{*}=\frac{\partial H_{\alpha}(\cdot)}{\partial \lambda_{2}}=-\frac{I_{\mathrm{sc}}\left(\mathrm{SOC}_{\mathrm{sc}}^{*}, I_{\mathrm{DC}, 0}^{*}\right)}{C_{\mathrm{sc}}}, \\
& \dot{\lambda}_{2}^{*}=-\frac{\partial H_{\alpha}(\cdot)}{\partial \mathrm{SOC}_{\mathrm{sc}}}=\frac{\lambda_{1}^{*}}{Q_{\mathrm{bat}, 0}} \frac{\partial I_{\mathrm{bat}}\left(\mathrm{SOC}_{\mathrm{bat}}^{*}\right)}{\partial \mathrm{SOC}_{\mathrm{sc}}}+\frac{\lambda_{2}^{*}}{C_{\mathrm{sc}}} \\
& . \frac{\partial I_{\mathrm{sc}}\left(\mathrm{SOC}_{\mathrm{sc}}^{*}, I_{\mathrm{DC}, o}^{*}\right)}{\partial \mathrm{SOC}_{\mathrm{sc}}} \ldots \\
& -2 \lambda_{d}^{*}\left[\mathrm{SOC}_{\mathrm{sc}}^{*}-\mathrm{SOC}_{\mathrm{sc}, \mathrm{min}}\right] \\
& \cdot \operatorname{sg}\left(\mathrm{SOC}_{\mathrm{sc}, \min }-\mathrm{SOC}_{\mathrm{sc}}^{*}\right) \ldots \\
& -2 \lambda_{d}^{*}\left[\mathrm{SOC}_{\mathrm{sc}, \max }-\mathrm{SOC}_{\mathrm{sc}}^{*}\right] \\
& \cdot \operatorname{sg}\left(\mathrm{SOC}_{\mathrm{sc}}^{*}-\mathrm{SOC}_{\mathrm{sc}, \max }\right) \text {, } \\
& \dot{X}_{d}^{*}=-\frac{\partial H_{\alpha}(\cdot)}{\partial \lambda_{d}}=\left[\mathrm{SOC}_{\mathrm{sc}}^{*}-\mathrm{SOC}_{\mathrm{sc}, \min }\right]^{2} \\
& \cdot \operatorname{sg}\left(\mathrm{SOC}_{\mathrm{sc}, \min }-\mathrm{SOC}_{\mathrm{sc}}^{*}\right)+\left[\mathrm{SOC}_{\mathrm{sc}, \max }-\mathrm{SOC}_{\mathrm{sc}}^{*}\right]^{2} \\
& \cdot \operatorname{sg}\left(\mathrm{SOC}_{\mathrm{sc}}^{*}-\mathrm{SOC}_{\mathrm{sc}, \max }\right) \text {, }
\end{aligned}
$$

$$
\begin{aligned}
& \operatorname{SOC}_{\mathrm{bat}}^{*}(T) \approx \mathrm{SOC}_{\mathrm{bat}}^{*}(0)=\mathrm{SOC}_{\mathrm{bat}, 0} ; \\
& \mathrm{SOC}_{\mathrm{bat}}^{*} \in\left[\mathrm{SOC}_{\mathrm{bat}, \mathrm{min}}, \mathrm{SOC}_{\mathrm{bat}, \mathrm{max}}\right], \\
& \mathrm{SOC}_{\mathrm{sc}}^{*}(\mathrm{~T}) \approx \mathrm{SOC}_{\mathrm{sc}}^{*}(0)=\mathrm{SOC}_{\mathrm{sc}, 0} ; \\
& X_{d}^{*}(0)=0, \\
& \frac{\mathrm{SOC}_{\mathrm{sc}}^{*} \in\left[\mathrm{SOC}_{\mathrm{sc}, \mathrm{min}}, \mathrm{SOC}_{\mathrm{sc}, \max }\right],}{\left.\partial \mathrm{SOC}_{\mathrm{sc}}\right)}=-\frac{V_{\mathrm{sc}, \max } I_{\mathrm{sc}}\left(\mathrm{SOC}_{\mathrm{sc}}\right)}{\sqrt{\left(\mathrm{SOC}_{\mathrm{sc}} V_{\mathrm{sc}, \mathrm{max}}\right)^{2}-4 R_{\mathrm{sc}} P_{\mathrm{sc}}}} \\
& \dot{\lambda}_{1}^{*}=0 ; \\
& \dot{\lambda}_{2}^{*}=0 ; \\
& \dot{\lambda}_{d}^{*}=0, \\
& \lambda_{1}^{*}=\lambda_{10} ; \\
& \lambda_{2}^{*}=\lambda_{20} ; \\
& \lambda_{d}^{*}=\lambda_{d 0}, \\
& H_{a}\left(\mathrm{SOC}_{\mathrm{bat}}^{*}, \mathrm{SOC}_{\mathrm{sc}}^{*}, T_{\mathrm{ice}}^{*}, I_{\mathrm{DC}, o}^{*}, \lambda_{1}^{*}, \lambda_{2}^{*}, \lambda_{d}^{*}\right) \\
& \leq H_{a}\left(\mathrm{SOC}_{\mathrm{bat}}^{*}, \mathrm{SOC}_{\mathrm{sc}}^{*}, T_{\mathrm{ice}}, I_{\mathrm{DC}, \mathrm{o}}, \lambda_{1}^{*}, \lambda_{2}^{*}, \lambda_{d}^{*}\right) ; \\
& \forall t \in[0, T] ; \forall\left(T_{\mathrm{ice}}, I_{\mathrm{DC}, o}\right) \in \Omega,
\end{aligned}
$$

where $\Omega=\left\{T_{\text {ice }} \in\left(T_{\text {ice, } \min }\left(\omega_{\text {ice }}(t), \overline{T_{\text {ice, } \max }}\left(\omega_{\text {ice }}(t)\right)\right)\right) ; I_{\mathrm{DC}, o} \in\right.$ $\left.\left(I_{\mathrm{DC}, o}, \overline{I_{\mathrm{DC}, o}}\right)\right\}$ is the capacities of control variable $T_{\text {ice }}$ and $\overline{I_{\mathrm{DC}, o}}$.

From (13)-(15) it shows that solving the optimal control problem is transformed into solving the initial conditions $\mathrm{SOC}_{\mathrm{bat}, 0}$ and $\mathrm{SOC}_{\mathrm{sc}, 0}$ of Li-SC HESS state of charge and costate variable initial value $\lambda_{0}=\left(\lambda_{10}, \lambda_{20}, \lambda_{d 0}\right)$. As SOC $\mathrm{Sat}, 0_{0}$, $\mathrm{SOC}_{\mathrm{sc}, 0}$ can be given directly; then it is further simplified into solving the initial output coefficients $\lambda_{10}$ and $\lambda_{20}$ of each energy storage element and the penalty intensity factor $\lambda_{d 0}$ under the constraints of the boundary condition in the vehicle driving cycle. Besides, the initial value of the costate $\lambda_{0}$ requires the minimum value of the control variables $T_{\text {ice }}$ and $I_{\mathrm{DC}, o}$ within the allowable range $\Omega$. So define $s_{1} \triangleq$ $-\lambda_{1} / E_{\text {bat }}\left(\mathrm{SOC}_{\mathrm{bat}}\right) \mathrm{Q}_{\mathrm{bat}, 0} ; s_{2} \triangleq-\lambda_{2} / \mathrm{SOC}_{\mathrm{sc}} C_{\mathrm{sc}}$, the Hamiltonian mathematical model of the system can be expressed as formula (16).

$$
\begin{aligned}
H_{a}\left(\mathrm{SOC}_{\mathrm{bat}}, \mathrm{SOC}_{\mathrm{sc}}, T_{\mathrm{ice}}, I_{\mathrm{DC}, o}, s_{1}, s_{2}\right) & \\
= & P_{\text {fuel }}\left(T_{\mathrm{ice}}, \omega_{\mathrm{ice}}\right)+s_{1} P_{\mathrm{bat}, i}\left(\mathrm{SOC}_{\mathrm{bat}}\right) \cdots \\
& \quad+s_{2} P_{\mathrm{sc}, i}\left(\mathrm{SOC}_{\mathrm{sc}}, I_{\mathrm{DC}, o}\right)+\lambda_{d} \Phi\left(\mathrm{SOC}_{\mathrm{sc}}\right) .
\end{aligned}
$$

From (16), $P_{\text {fuel }}\left(T_{\text {ice }}, \omega_{\text {ice }}\right), P_{\text {bat }, i}\left(\mathrm{SOC}_{\mathrm{bat}}\right)$, and $P_{\mathrm{sc}, i}\left(\mathrm{SOC}_{\mathrm{sc}}\right.$, $\left.I_{\mathrm{DC}, o}\right)$, respectively, correspond to ICE fuel consumption power, internal lithium-ion battery power, and SC power at current time, so it is the same with $s_{1}, s_{2}$, and $\lambda_{d}$ as the weighting factor of Li-SC HESS. It is apparent that the practical significance of Hamiltonian function is described as 
the equivalent fuel power function, it is also to be the sum of PHEV weighted power within a certain drive period, which is consistent with the law of conservation of energy. So it verifies the feasibility of PMP algorithm in the application of the actual object. So PMP algorithm can be transformed into an online " $\lambda$-control" method under the optimal solution of minimum fuel consumption.

4.3. " $\lambda$-Control" Based PSO-PI Real-Time Optimization. Although the costate variable initial value $\lambda_{0}$ is a constant in off-line, HESS output power differs from different cycle driving conditions in drive cycle. So using the PMP algorithm is difficult to ensure the real-time characteristic of " $\lambda$-control." In order to respond to the online noncausal optimal control strategy, this research uses PSO (Particle Swarm Optimization) algorithm to optimize the parameters of PI closed-loop controller (PSO-PI controller), which is to improve the characteristics of flexibility and adaptability of feedback closed-loop controller, besides its robustness and fast convergence speed, easily implementing and high computational efficiency.

Considering $\mathrm{SOC}(t)$ of each energy storage unit of $\mathrm{Li}$ SC HESS, the research assumes that the reference value of Li-SC HESS SOC $(t)$ is $\mathrm{SOC}_{\text {ref }}$, under the computer control system environment; set the sampling period $T$ and $t=$ $k T$ and introduce the PI feedback closed-loop equation (17), corresponding to the PSO-PI control block diagram (Figure 9).

$$
\begin{aligned}
\tilde{\lambda}(t)= & \lambda_{0}+k_{p}\left(\mathrm{SOC}_{\mathrm{ref}}-\operatorname{SOC}(t)\right) \\
& +k_{i} \sum_{i=1}^{k}\left(\mathrm{SOC}_{\mathrm{ref}}-\operatorname{SOC}(i)\right) .
\end{aligned}
$$

From (17), there are two parameters $k_{p}$ and $k_{i}$ of PI controller to be optimized. According to the ITAE (integral of time multiplied by the absolute value of error) indicators, considering the steady-state error, the performance of settling time, small overshoot, and oversmooth, it uses criterion ITAE of PSO algorithm to calculate the objective function. In addition, every potential optimal solution of optimization problems to be optimized in PSO algorithm represents a particle in one of the solvable space, such as particle $i$ which corresponds to the fitness value of $i$-particle fitness function. This research introduces the particle current position $x_{i}=\left(x_{i 1}, x_{i 2}, \ldots, x_{i d}\right), i=1,2, \ldots, n$, current speed $v_{i}=$ $\left(v_{i 1}, v_{i 2}, \ldots, v_{i d}\right)$, all particles' best flying position trajectory $P_{i}=\left(p_{i 1}, p_{i 2}, \ldots, p_{i d}\right)$, monomer extreme value $p_{\text {best }, i}=$ $\left(p_{\text {best }, i 1}, p_{\text {best }, i 2}, \ldots, p_{\text {best }, i d}\right)$, group extreme value $p_{\text {gbest }, i}=$ $\left(p_{\text {gbest }, i 1}, p_{\text {gbest }, i 2}, \ldots, p_{\text {gbest }, i d}\right)$, and inertia weight $h$ and then updates and iterates the particle according to formula (18).

$$
\begin{aligned}
J= & \int_{0}^{+\infty} t|e(t)| d t \\
v_{i d, k+1}= & h v_{i d, k}+c_{1} r_{1} \times\left(p_{\text {best }, i d, k}-x_{i d, k}\right)+c_{2} r_{2} \\
& \times\left(p_{\text {gbest }, i d, k}-x_{i d, k}\right),
\end{aligned}
$$

$$
\begin{aligned}
x_{i d, k+1} & =x_{i d, k}+v_{i d, k+1}, \\
h & =h_{\text {initial }}-\frac{\left[\left(h_{\text {initial }}-h_{\text {end }}\right)\right] * k}{k_{\max }} .
\end{aligned}
$$

From (18), $d=1,2, \ldots, D ; h$ is the inertia weight; $r_{1}$ and $r_{2}$ are the random numbers between $(0,1) ; c_{1}$ and $c_{2}$ are the nonnegative constant evolution factor; $x_{i d, k}$ and $v_{i d, k}$ are the updated position and speed of particle $i$ at the $k$ th iteration among $D$-dimensional space; $h_{\text {initial }}$ is the initial inertia weight; $k_{\max }$ is the maximum number of iterations; $h_{\text {end }}$ is the inertia weight when $k_{\max }$. Taking $h_{\text {initial }}=0.9$ and $h_{\text {end }}=0.4$ to ensure a strong initial global search capability, the latter part of the algorithm facilitates local search.

The main steps of PSO-PI controller parameter optimization are as follows:

(1) Assuming that the particle $i$ has parameters $k_{p}, k_{i}$, group scale, current iteration number $k$ and the iteration maximum number $k_{\text {max }}$, inertia weight, learning factor, monomer extreme value $p_{\text {best }, i}$, and group extreme value $p_{\text {gbest }, i}$, and so on, then initialize $x_{i}$ and $v_{i}$ of particle $i$ randomly.

(2) Update the $x_{i d, k}$ and $v_{i d, k}$ of particle $i$ according to formula (18) and then calculate its fitness value $J_{i}$.

(3) Compare $J_{i}$ with the corresponding $p_{\text {best }, i}$; if $J_{i}>$ $p_{\text {best }, i}$, update the position of $p_{\text {best }, i}$ instead of the current position of $P_{i}$.

(4) Similarly, compare $J_{i}$ with the corresponding $p_{\text {gbest }, i}$; if $J_{i}>p_{\text {gbest }, i}$, update the position of $p_{\text {gbest }, i}$ instead of the current position of $P_{i}$.

(5) Judge the termination constraints of PSO algorithm; if it is terminated, go directly to step (6); otherwise, repeat steps (2)-(4).

(6) Output the optimized parameter values $k_{p}$ and $k_{i}$.

Since PI controller is not adaptive itself, add PSO algorithm to adjust the parameters of the controller; the selfadaptability is improved to achieve the purpose of fast tracking and controlling the covariable in PMP algorithm. When group scale is set to 30 , the maximum calculation period to is set to 100 , and both $c_{1}$ and $c_{2}$ are set to 1.50425 , the convergence curve of the best individual fitness function is shown in Figure 10.

4.4. Management of Li-SC HESS Limited Power. Above, the research takes the vehicle dynamic character and minimal fuel consumption as the main analysis object and initially establishes energy optimization management method of PHEV power system. Although the allocated processing factors can ensure the coordinated allocation of power between lithium-ion battery and SC, SOC constraints during controlling just to prevent Li-SC HESS overcharge and overdischarge, which are the basic conditions. In order to further improve Li-SC HESS performance, it needs to make a real-time management of the power of Li-SC HESS each energy storage unit during the charging/discharging state. 


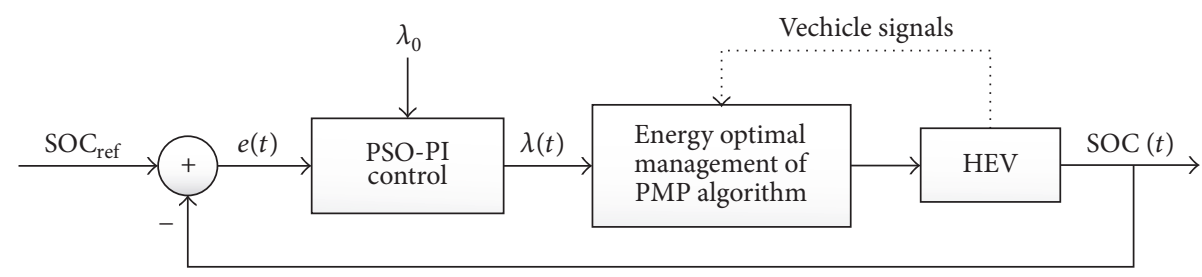

FIGURE 9: PSO-PI real-time optimization “ $\lambda$-control” block diagram.

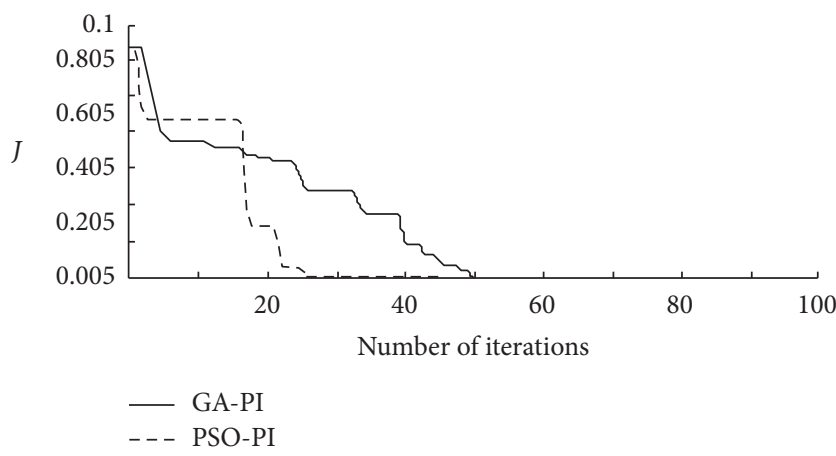

FIGURE 10: The convergence curve of the best individual fitness function.

The vehicle energy optimization control flow chart is shown in Figure 11.

According to the lithium-ion battery's characteristics of low power density, strong energy density, and limited life, the research adopts the preresponse principle of SC when the demanded power of the alternator changes and dictates that when $\mathrm{SOC}_{\mathrm{sc}}$ of SC reaches the limiting value of serious charge/overcharge, Li-SC HESS prohibits its charge/discharge. When $\mathrm{SOC}_{\mathrm{sc}}$ has not reached serious critical limits, it will be divided into the normal working section $\left(\mathrm{SOC}_{\text {low }}, \mathrm{SOC}_{\text {high }}\right.$ ) and power limitation management section $\left(\mathrm{SOC}_{\text {high }}, \mathrm{SOC}_{\max }\right) \cup\left(\mathrm{SOC}_{\text {min }}, \mathrm{SOC}_{\text {low }}\right)$. That is as follows.

$\Delta P_{\text {bat }}$ and $\Delta P_{\text {sc }}$ are the amended power of lithium-ion battery and SC, respectively, and there is $\Delta P_{\text {bat }}=-\Delta P_{\mathrm{sc}}$. During the normal operation, $\mathrm{SOC}_{\mathrm{sc}} \in\left(\mathrm{SOC}_{\text {low }}, \mathrm{SOC}_{\text {high }}\right)$, $\Delta P_{\mathrm{sc}}=0$, and the power of each storage device does not change. When the discharging power exceeds the limit, the rule of power correction is shown in formula (19). Similarly, there is formula (20) when the charging power exceeds the limit.

$$
\begin{aligned}
& \Delta P_{\mathrm{sc}}=0, \quad \mathrm{SOC}_{\mathrm{sc}}>\mathrm{SOC}_{\mathrm{sc} \_ \text {max }}, \\
& \Delta P_{\mathrm{sc}}=P_{\mathrm{sc} \_ \text {ref }}\left(\frac{\mathrm{SOC}_{\mathrm{sc}}-\mathrm{SOC}_{\mathrm{sc} \text { high }}}{\mathrm{SOC}_{\mathrm{sc} \_ \text {max }}-\mathrm{SOC}_{\mathrm{sc} \_ \text {high }}}\right)=A, \\
& \mathrm{SOC}_{\mathrm{sc}} \in\left(\mathrm{SOC}_{\mathrm{sc} \_ \text {high }}, \mathrm{SOC}_{\mathrm{sc} \_ \text {max }}\right), \\
& \Delta P_{\mathrm{sc}}=-P_{\mathrm{sc} \text { ref }}\left(\frac{\mathrm{SOC}_{\mathrm{sc} \_ \text {low }}-\mathrm{SOC}_{\mathrm{sc}}}{\mathrm{SOC}_{\mathrm{sc} \_ \text {low }}-\mathrm{SOC}_{\mathrm{sc} \_ \text {min }}}\right)=B, \\
& \mathrm{SOC}_{\mathrm{sc}} \in\left(\mathrm{SOC}_{\mathrm{sc} \_ \text {min }}, \mathrm{SOC}_{\mathrm{sc} \_ \text {low }}\right), \\
& \Delta P_{\mathrm{sc}}=-P_{\mathrm{sc} \_ \text {ref }}, \quad \mathrm{SOC}_{\mathrm{sc}}<\mathrm{SOC}_{\mathrm{sc} \_ \text {low }}
\end{aligned}
$$

$$
\begin{aligned}
& \Delta P_{\mathrm{sc}}=-P_{\mathrm{sc} \_ \text {ref }}, \quad \mathrm{SOC}_{\mathrm{sc}}>\mathrm{SOC}_{\mathrm{sc} \_ \text {max }}, \\
& \Delta P_{\mathrm{sc}}=-A, \quad \mathrm{SOC}_{\mathrm{sc}} \in\left(\mathrm{SOC}_{\mathrm{sc} \_ \text {high }}, \mathrm{SOC}_{\mathrm{sc} \_ \text {max }}\right), \\
& \Delta P_{\mathrm{sc}}=-B, \quad \mathrm{SOC}_{\mathrm{sc}} \in\left(\mathrm{SOC}_{\mathrm{sc} \_ \text {min }}, \mathrm{SOC}_{\mathrm{sc} \_ \text {low }}\right), \\
& \Delta P_{\mathrm{sc}}=0, \quad \mathrm{SOC}_{\mathrm{sc}}<\mathrm{SOC}_{\mathrm{sc} \_ \text {low }} .
\end{aligned}
$$

\section{Results and Analysis}

PHEV with Li-SC HESS in this paper is obtained by the secondary development of PHEV model based on ADVISOR software (Figure 12).

Taking into account of the majority of domestic small car users daily and mainly using in the city, the research adopts the urban road cycling conditions (CYC-UDDS). The realtime curve of driving cycle speed is shown in Figure 13(a). The gear position curve of the driving cycle is shown in Figure 13(b). Integrating Figures 13(a) and 13(b), it can be seen that the vehicle speed of the driving cycle is welltracked with gear position changes, which basically meets the evaluation requirements of vehicles driving cycle actually. It also verifies the feasibility of energy-optimized controlling electric vehicles by PMP algorithm.

(1) The comparison results of PHEV power system before and after: when working normally in the vehicle driving cycle, Li-SC HESS can provide or absorb some of the energy through alternator, reducing fuel consumption ICE. Figures 14(a) and 14(b), respectively, represent the alternator torque curve of vehicle power system, controlled by PMP energy optimization algorithm.

Comparing Figure 14(a) with Figure 14(b), it is easy to know that, after using PMP algorithm, the alternator torque curve fluctuation changes more dramatically than before and the alternator power requirements increase significantly. According to the conservation of energy, it indicates that the part of ICE fuel consumption absorbed by alternator increases clearly.

(2) The result of real-time optimization of output coefficients by PSO algorithm: in order to demonstrate the characteristic of real-time tracking by PSO-PI controller, the research obtains the output coefficient $\lambda(t)$ curve (Figure 15) of Hamiltonian function under the action of single lithiumion battery.

From Figure 15, this control strategy can also adjust ICE to moving along its track of minimum fuel energy consumption through the alternator real-timely, and it also further validates the rationality and feasibility of PMP algorithm. 


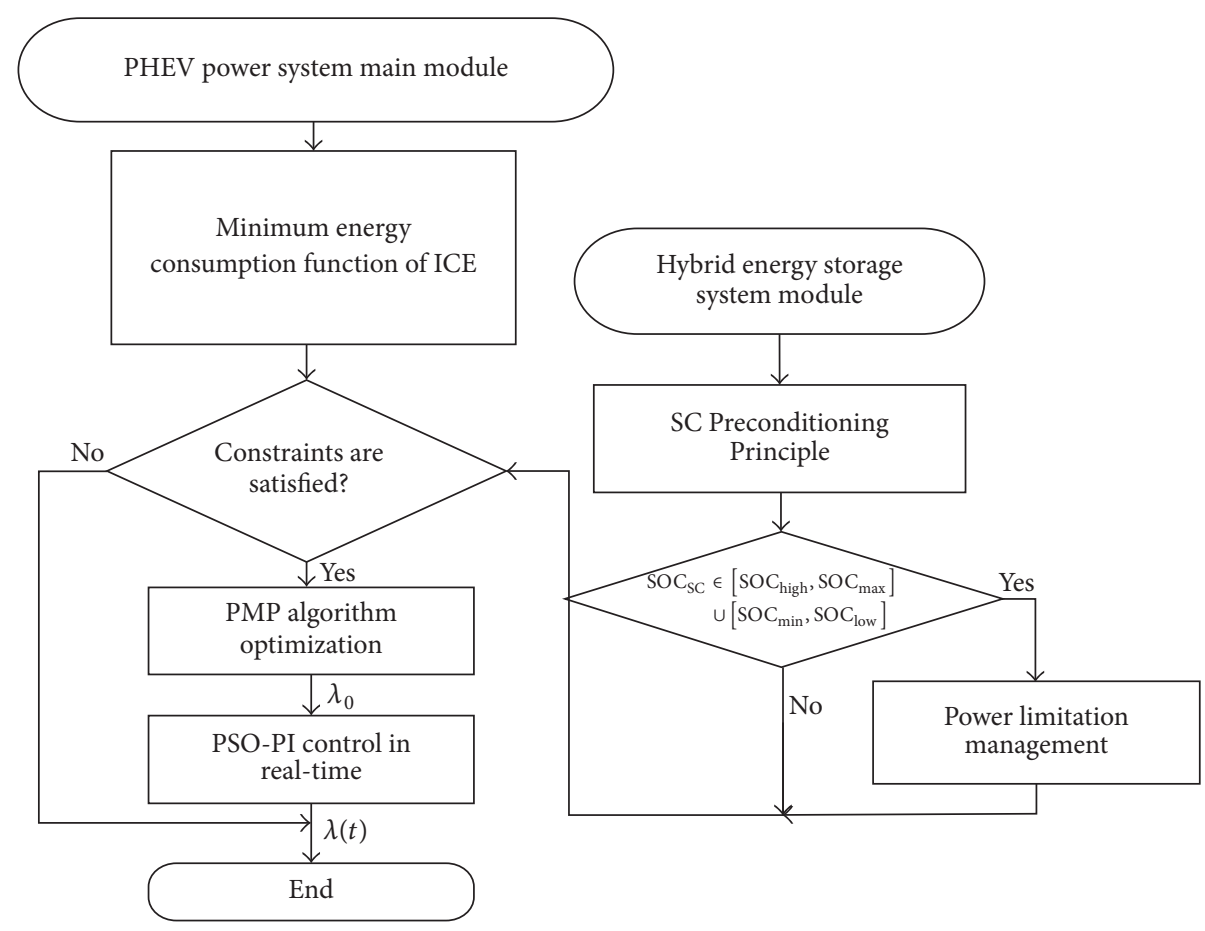

FIGURE 11: The overall flow chart of PHEV energy optimization control.

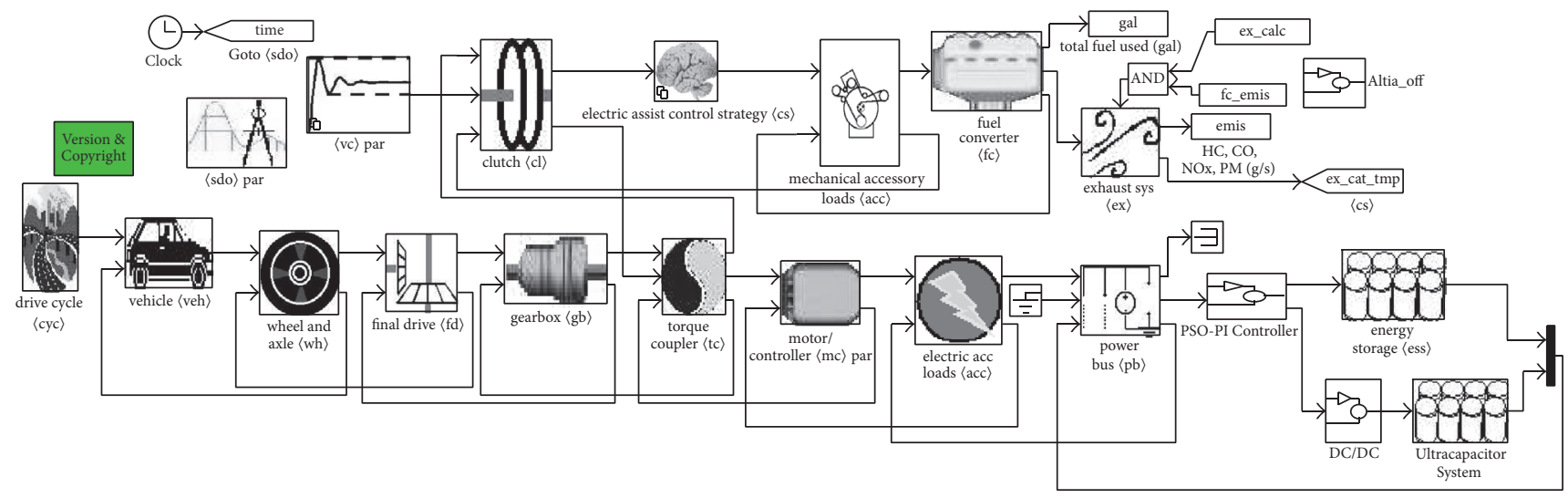

FIGURE 12: PHEV simulation model.

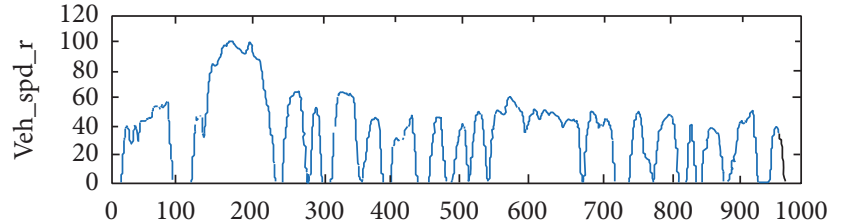

— Veh_spd_r

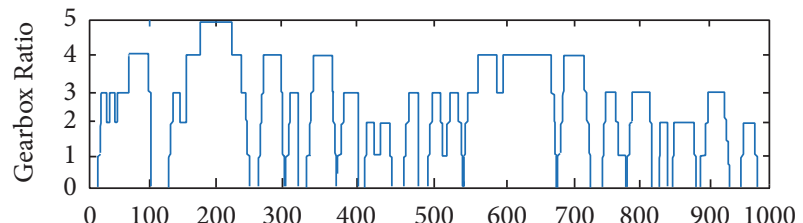

- Gearbox ratio

(b)

FIGURE 13: (a) Under CYC-UDDS, real-time curve of driving cycle speed. (b) Under CYC-UDDS, gear position curve of the driving cycle. 


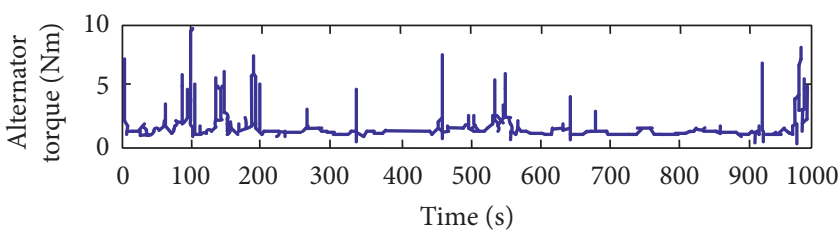

(a)

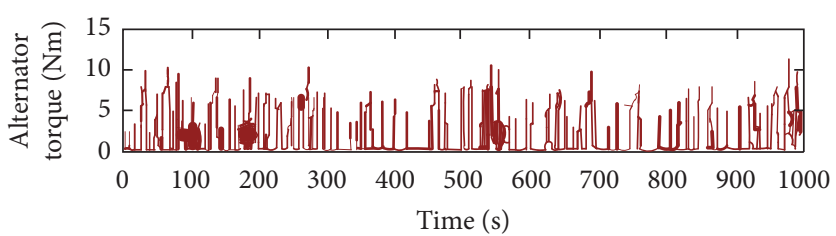

(b)

FIgURe 14: (a) Before PMP energy optimization algorithm. (b) After PMP energy optimization algorithm.

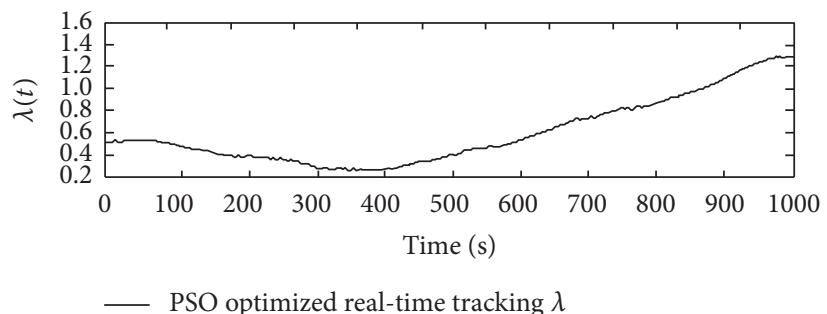

FIGURE 15: Output coefficient curve of real-timely optimized Hamiltonian function by PSO-PI controller.

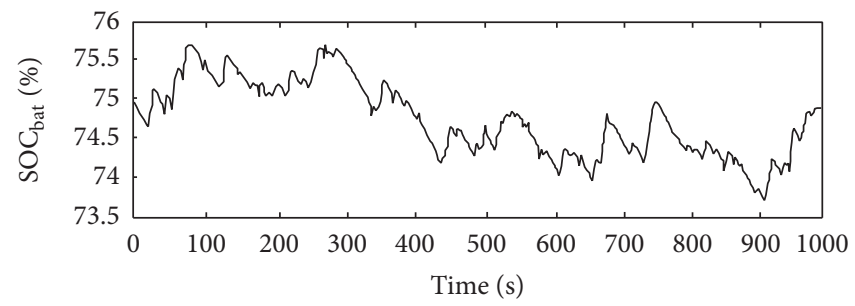

(a)

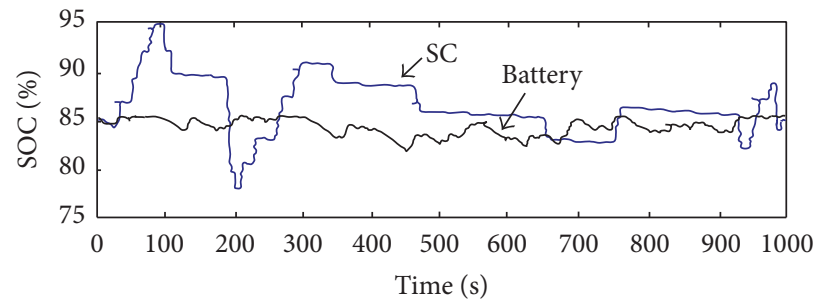

(b)

FIGURE 16: (a) SOC curve of a single lithium-ion battery energy storage. (b) SOC curve of Li-SC HESS.

The simulation situation between the single lithium-ion battery energy storage and Li-SC HESS. It can be known from Figures 16(a) and 16(b) that the state of charge of lithium battery or SC is consistent with the end constraints of Pontryagin's minimum principle. Besides, as is shown in Figures $17(\mathrm{a})$ and $17(\mathrm{~b})$ together with Figure 16(b), since LiSC HESS is embedded with SC in the vehicle driving cycle, it can significantly reduce the output of lithium-ion battery. The charge and discharge currents of lithium-ion battery are clearly smaller than the single lithium-ion batteries, which not only well smooth the charging/discharging process of batteries, but also obviously reduce the corresponding lithiumion batteries' charging/discharging cycle times. Finally, it reflects the good coordinate ability between Li-SC HESS each energy storage element, which can extend battery's life. And it also validates the validity and effectiveness of this energyoptimized control method for designed PHEV with Li-SC HESS.

\section{Summary}

The research designs a kind of PHEV that introduces Li-SC HESS. Compared with traditional vehicles, This PHEV has the advantages of both ICE and Li-SC HESS. For example, the internal ICE can use existing gas station resources and reduce the overall investment costs. Meanwhile, it can alleviate the difficulty more effectively than pure electric vehicles when solving the problems brought from defrosting, air conditioners, and other pieces of large energy consumption equipment. Li-SC HESS can help extend battery life and extend the driving ranges of cars. In particular, the embedment of SC makes Li-SC HESS well suited to start the vehicle, the speed change, and energy recovery during braking. Mainly, PHEV energy optimization control strategy can effectively reduce vehicle exhaust emissions, benefit for the urban environment, which has a high research value.

\section{Competing Interests}

The authors declare that they have no competing interests.

\section{Acknowledgments}

The research group would like to thank the "Research on Electric Vehicle Li-ion battery and SC Hybrid Energy Storage System Energy Management Strategy" (Grant no. 51677058) for funding this research. 


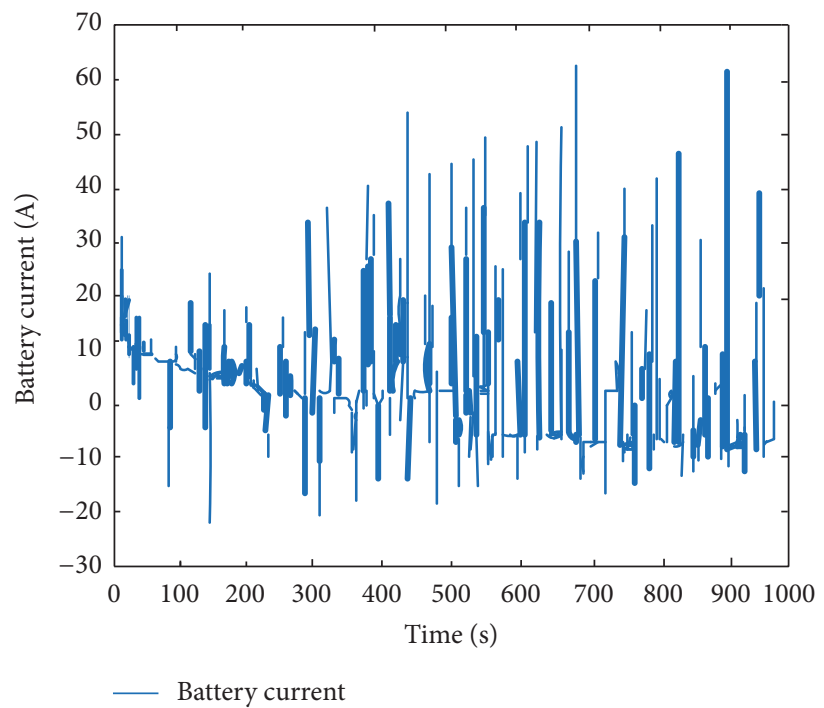

(a)

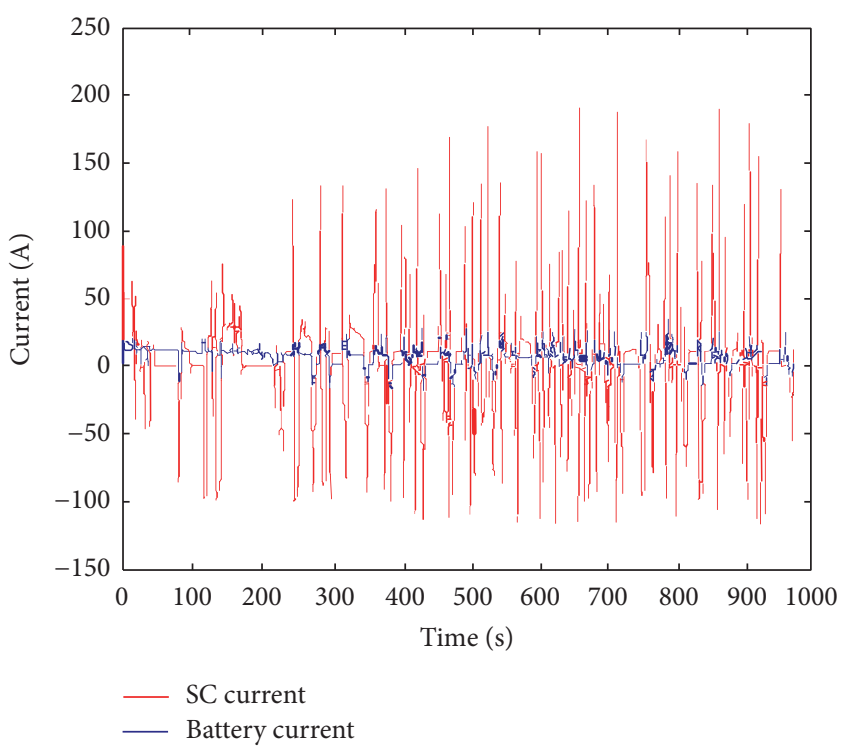

(b)

FIGURE 17: (a) Current curve of a single lithium-ion battery energy storage. (b) Current curve of Li-SC HESS.

\section{References}

[1] A. Santucci, A. Sorniotti, and C. Lekakou, "Power split strategies for hybrid energy storage systems for vehicular applications," Journal of Power Sources, vol. 258, pp. 395-407, 2014.

[2] M. Masih-Tehrani, M.-R. Ha'iri-Yazdi, V. Esfahanian, and A. Safaei, "Optimum sizing and optimum energy management of a hybrid energy storage system for lithium battery life improvement," Journal of Power Sources, vol. 244, pp. 2-10, 2013. 


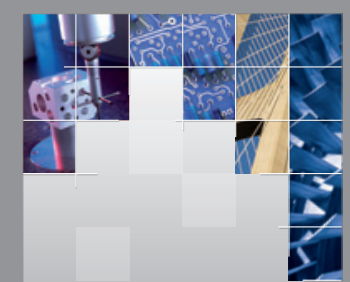

\section{Enfincering}
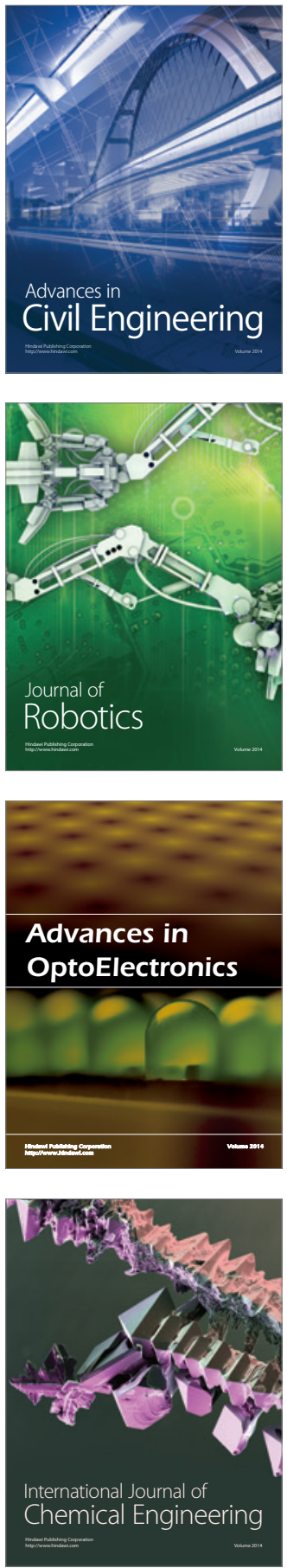

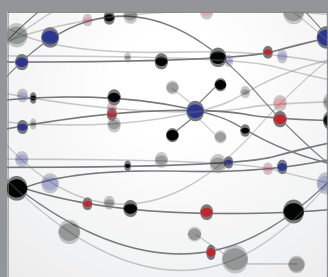

The Scientific World Journal

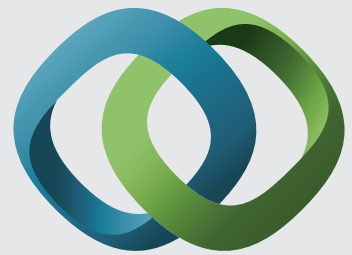

\section{Hindawi}

Submit your manuscripts at

https://www.hindawi.com
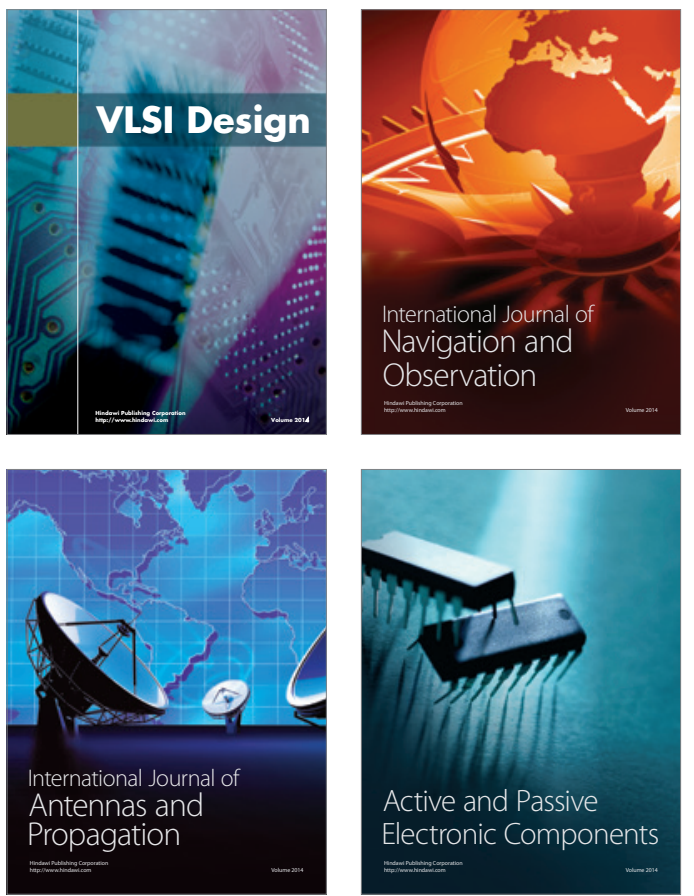
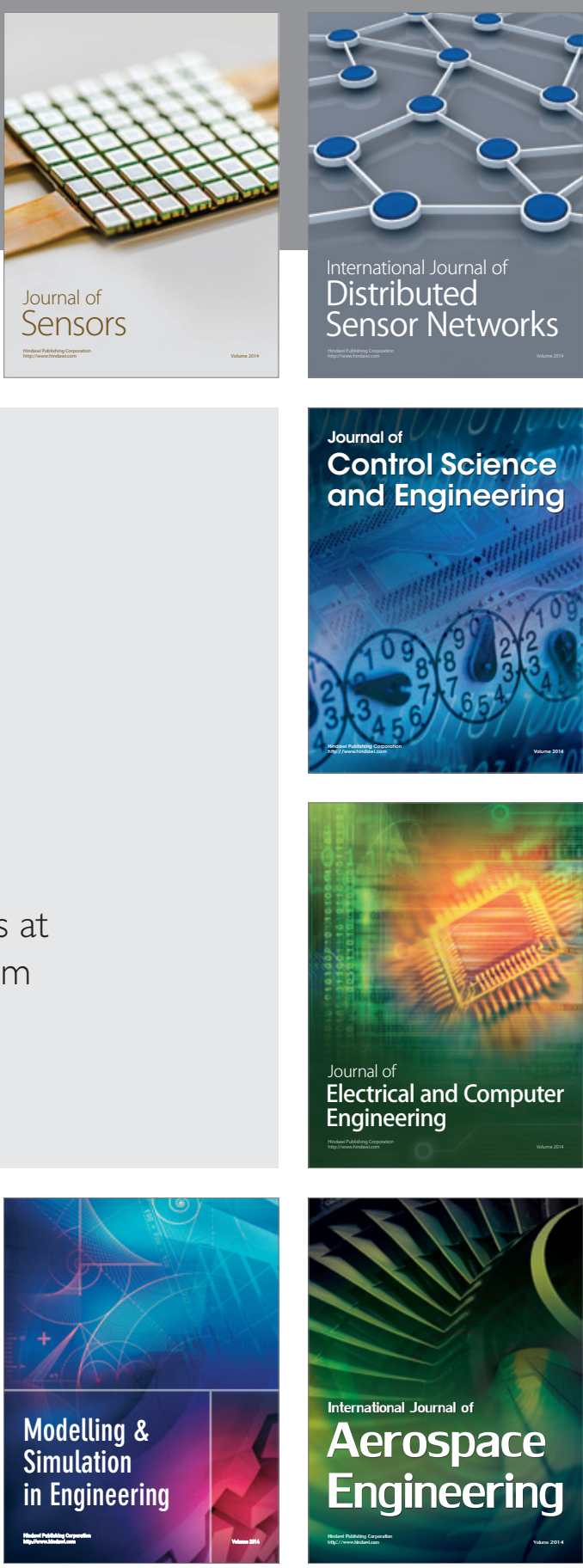

International Journal of

Distributed

Sensor Networks

$-$

Joumal of

Control Science

and Engineering
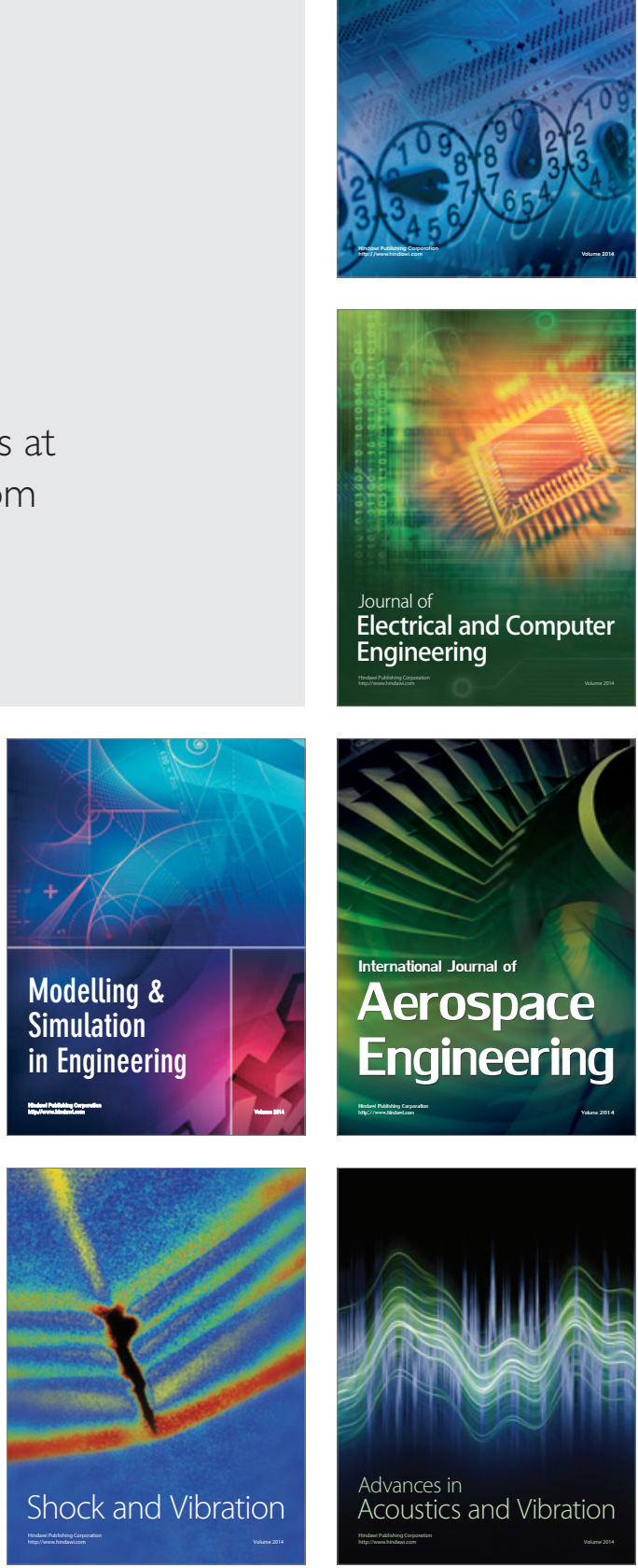